\title{
Therapeutic proton irradiation results in apoptosis and caspase-3 activation in human peripheral blood lymphocytes
}

\author{
Justyna Miszczyk ${ }^{1}$, Kamila Rawojć ${ }^{2}$, Anna Maria Borkowska ${ }^{1}$, Agnieszka Panek ${ }^{1}$, Jan Swakoń ${ }^{1}$, Aleksander \\ Gałaś $^{3}$, Mansoor M. Ahmed ${ }^{4}$, Pataje G. S. Prasanna ${ }^{4}$ \\ ${ }^{1}$ Institute of Nuclear Physics Polish Academy of Sciences, Kraków, Poland; ${ }^{2}$ Department of Endocrinology, Nuclear Medicine Unit, The University \\ Hospital, Kraków, Poland; ${ }^{3}$ Department of Epidemiology, Jagiellonian University Medical College, Kraków, Poland; ${ }^{4}$ Radiation Research Program, \\ National Cancer Institute, National Institutes of Health, Bethesda, Maryland, USA \\ Contributions: (I) Conception and design: J Miszczyk; (II) Administrative support: J Miszczyk; (III) Provision of study materials or patients: J Miszczyk, \\ A Gałaś; (IV) Collection and assembly of data: A Panek, J Miszczyk, K Rawojć; (V) Data analysis and interpretation: MM Ahmed, PG Prasanna, K \\ Rawojć, J Miszczyk; (VI) Manuscript writing: All authors; (VII) Final approval of manuscript: All authors. \\ Correspondence to: Justyna Miszczyk. Institute of Nuclear Physics Polish Academy of Sciences, Kraków PL-31342, Poland. \\ Email: justyna.miszczyk@ifj.edu.pl.
}

\begin{abstract}
Background: Proton therapy is effective in controlling many cancer types, allowing to spare the normal tissues and limiting the risk of adverse effects. However, cellular and molecular mechanisms by which protons induce cell death are still not fully understood. The purpose of this study was to investigate apoptotic mode of cell killing in human peripheral blood lymphocytes (HPBL) exposed ex vivo to $60 \mathrm{MeV}$ proton beam radiation.
\end{abstract}

Methods: HPBL obtained from 5 healthy donors were irradiated ex vivo with $60 \mathrm{MeV}$ protons in spread out Bragg peak (SOBP), in the dose range 0.3-4.0 Gy. The average proton dose rate was 0.075 Gy/s. After irradiation, HPBL were stained with Annexin V fluorescein isothiocyanate (V-FITC) at different time-points post-radiation exposure: 1, 4 and 24 hours. To assess caspase- 3 activation following irradiation with protons, caspase-3 DEVD-R1100 Fluorometric assay was used.

Results: The apoptotic cell fraction stained with Annexin V-FITC, analysed after 1 and 4 h post protonirradiation showed a dose-response increase in cell death. After $24 \mathrm{~h}$ post radiation exposure, the apoptotic fraction of cells represented a similar trend as in 1 and $4 \mathrm{~h}$ but less pronounced. Caspase- 3 activation measured after $6 \mathrm{~h}$ of proton irradiation was significantly higher $(\mathrm{P}<0.05)$ compared to $24 \mathrm{~h}$ post-irradiation for all donors.

Conclusions: The data clearly demonstrates that $60 \mathrm{MeV}$ therapeutic proton beam induced cell-killing in HPBL via apoptotic cell mode of death appears to be mediated by caspase- 3 activation.

Keywords: Human peripheral blood lymphocytes (HPBL); cell-killing; apoptosis; proton therapy; radiotherapy; Annexin V-FITC; caspase-3

Submitted Apr 18, 2018. Accepted for publication Jun 13, 2018.

doi: $10.21037 /$ tcr.2018.06.14

View this article at: http://dx.doi.org/10.21037/tcr.2018.06.14

\section{Introduction}

Protons are used in radiation therapy to maximize tumour control with minimal normal tissue complication in order to obtain maximum therapeutic gain $(1,2)$. Spectacular technological developments over the last decade and establishment of new proton centres around the world are likely to result in an increase in the number of patients treated with protons for certain tumour types, including paediatric tumours $(2,3)$. It is generally accepted that protons are radiobiologically more effective in cell-killing 
compared to $\mathrm{X}$-rays $(3,4)$ and produce a higher frequency of cytogenetic damage in human peripheral blood lymphocytes (HPBL), when exposed ex vivo (4).

Studies on the differences of the mechanisms of cellular injury by protons compared to $\mathrm{X}$-rays are imperative to fully understand therapeutic implications, potential normal tissue complications and develop suitable mitigation approaches. A better understanding of the effects of proton therapy at the molecular, cellular and tissue levels is necessary for further improving the efficiency of this emerging treatment option. Immune-related or redox-related biomarkers might help patients with cancer evaluation and providing insight into improved combination of radiotherapy with biological treatments for each individual (5).

HPBL is a well-accepted model to study the effects of radiation on normal tissue. HPBL are predominantly in a resting phase $\left(G_{0}\right)$ of the cell cycle; they are a synchronous and homogeneous cell population, which is in continuous trafficking throughout the body, and therefore, represent normal tissue. Circulating lymphocytes are involved in many key mechanistic roles following exposure to radiation therapy of tumours, which include, systemic responses at distant sites, enhancement of antitumour innate and adaptive immune response, enhanced tumour cell recognition and killing via up-regulation of antigen presenting machinery and induction of positive immunomodulatory pathways as they penetrate tumour microenvironment (6). Lymphocytes show higher propensity to apoptosis, which seems to depend on p53 pathway (7). Previously, we characterized the response of HPBL to therapeutic proton radiation of $60 \mathrm{MeV}$ by studying the nuclear division index, DNA damage, cell viability and compared it with $\mathrm{X}$-rays (4). A spatial difference in the energy deposition with protons compared to X-rays results in a localized intracellular manifestation of cytogenetic damage causing cell-killing (4). After radiation exposure, cells undergo different types of cell death, which includes apoptosis and necrosis (8). Extending our previous study (4), in our most recent work, using Apoptotic, Necrotic and Healthy Cells Quantification Kit (Biotium, Inc., Hayward, USA), we demonstrated that X-rays and protons induce cell-killing by apoptosis as well as necrosis, and protons are more efficient in cell-killing via necrosis compared to X-rays (9). Our studies also indicated that there might be differences among individuals in their response to these two radiation types (9), which may be the reason for the clinically observed type and intensity of adverse effects after proton therapy. Differences in cellkilling modes may have implications on the potential of a given therapeutic modality to cause immune modulation via apoptosis or necrotic cell death (10).

Fluorescein isothiocyanate (FITC)-labelled Annexin V (Annexin V-FITC) method is a reliable quantitative method to detect apoptosis. The mechanism behind apoptotic cells detection is based on the principle that during apoptosis phosphatidylserine (PS) is relocated from the inner to outer surface of the cell membrane and Annexin V-FITC can detect cells in which PS is localized on the outer cell membrane because of its high affinity for PS by green fluorescence signal (11-13). Due to the difficulties in detection of late apoptotic cell death and distinguishing it from necroptosis, various cell death molecular pathways of apoptosis are used to study this mode of cell-killing. The extrinsic and intrinsic cell death pathways converge at caspase-3, which together with its effector caspases, orchestrates dismantling of cell structure through cleavage of specific substrates (11). Thus, there is a massive caspase activation (cysteine aspartic acid-specific proteases), which is specific to apoptosis and a key event responsible for apoptosis progression (11) that includes activation of downstream effector caspases in the caspase cascade: caspase-3, caspase-6, and caspase-7 (11,14). Caspase-3 (CPP32, Yama or apopain) is recognized as one of most significant executioners (15). In contrast to apoptosis, necrosis is largely independent of caspase activity $(16,17)$. Caspase- 3 is a downstream effector caspase in the cascade $(15,18)$. Therefore, we were interested in studying the role of caspase- 3 activation in proton irradiation induced apoptosis in HPBL.

In this article, we present the results of our studies on apoptosis in HPBL following ex vivo irradiation with protons at different doses among 5 healthy donors. Early and late apoptotic cells were assessed using Annexin V-FITC staining and caspase-3 activity was measured at different post-irradiation time points, respectively. In addition, interindividual differences in apoptosis and caspase- 3 activation were also studied. Understanding the effect of proton irradiation on the activation of apoptotic pathway in HPBL, which represents normal tissue, may lead to the discovery of novel biomarkers of radiation injury, allow stratification of patients based on a risk of toxicity, allow selection of a patient for a specific type of radiation treatment $v s$. other, and/or use of radioprotector or a mitigator to enhance the therapeutic benefit. 


\section{Methods}

\section{Blood collection}

Whole peripheral blood was collected by phlebotomy in vacutainers containing lithium heparin from 5 healthy non-smoking donors ( 3 males and 2 females, age between 36-56 years) by the specialized company 'Diagnostics' in the laboratory of the Institute of Nuclear Physics, Kraków, Poland. Prior to blood collection, informed consent was obtained as described earlier (4). The human bioethical committee of the Regional Medical Board in Krakow approved the informed consent form used in this study (No. 124/KBL/OIL/2013).

\section{Lymphocytes isolation}

Lymphocytes were isolated from the whole blood by density gradient separation using Histopaque ${ }^{\circledR}-1077$ (SigmaAldrich, St. Louis, USA). Cells were checked for viability by the trypan blue exclusion test. For all donors, $99-100 \%$ of cells were dye-excluding.

\section{Proton irradiations and dosimetry}

Isolated lymphocytes in RPMI 1640 culture medium (PAA Laboratories GmbH, Pasching, Austria) were irradiated with proton beam in $2 \mathrm{~mL}$ Eppendorf vials (Eppendorf, Hamburg, Germany) at the Proton Therapy facility, Institute of Nuclear Physics Polish Academy of Sciences, Kraków, Poland (IFJ PAN). During irradiation, vials were placed into a specially designed Poly(methyl methacrylate) (PMMA) phantom, which was located at the irradiation setup isocentre [in the middle of spread out Bragg peak (SOBP)] and in the centre of the flat beam ( $40 \mathrm{~mm}$ broad, fully modulated proton field with a range of $30 \mathrm{~mm}$ ). All procedures have been previously described in detail $(4,19)$. HPBL at a concentration of $2 \times 10^{4}$ cells $/ \mathrm{mL}$ were irradiated ex vivo with doses of $0.3,0.5,0.75,1.0,1.5,2.0$, 2.5, 3.0, and 4.0 Gy (the average dose rate was $0.075 \mathrm{~Gy} / \mathrm{s}$ ). The proton beam dosimetry was done according to the TRS-398 protocol recommended by International Atomic Energy Agency (20) using a reference dosimeter consisting of a PTW TM31010 SemiFlex ionization chamber and a PTW UNIDOS Webline Electrometer (PTW, Freiburg, Germany). The dosimeter set was calibrated at the IFJ PAN at Theratron 780 Co-60 treatment unit. Dose measurements were performed in the middle of fully modulated SOBP and the overall uncertainty was less than
$3 \%$ with the precision of dose delivery greater than $0.5 \%$.

Proton irradiation was performed in ambient conditions and immediately after irradiation cells were put to a box with ice water and transported for the further processing. After irradiation, cells were incubated at $37^{\circ} \mathrm{C}$ in RPMI 1640 culture medium supplemented with $20 \%$ heatinactivated foetal bovine serum (Gibco, Carlsbad, USA). Non-irradiated lymphocytes (0 Gy) were considered as a biological control.

\section{Quantification of apoptosis by Annexin V-FITC binding assay}

In order to quantify apoptosis in ex vivo HPBL model after 1, 4 and $24 \mathrm{~h}$ following proton irradiation, Apoptotic, Necrotic and Healthy Cells Quantification Kit (Biotium, Inc.) was used. Briefly, during the experimental procedure after incubation time, HPBL were washed in PBS and resuspended in $1 \times$ Binding Buffer. Following, $5 \mu \mathrm{L}$ of FITC-Annexin $\mathrm{V}$ and $5 \mu \mathrm{L}$ of Hoechst 33342 solutions were added to each tube and incubated for $15 \mathrm{~min}$ at $21^{\circ} \mathrm{C}$ in the dark. Subsequently, cells were washed 2 times with $1 \times$ Binding Buffer, fixed with $2 \%$ formamide and placed on a glass slide covered with a glass coverslip. A total of $2 \times 10^{5}$ cells were analysed for each donor and dose of ionizing radiation.

Generally, 4-6 representative fields of at least 100 cells per dose, per time point were analyzed separately from 3 independent repetitions (slides), by 2 independent scorers, using the fluorescent microscopy coupled to an image analysis system (MetaSystems ${ }^{\mathrm{TM}}$, Altlussheim, Germany), according to the criteria described by Zhang et al. (12). All slides were coded and blinded to scorers. Sample decoding was done after completing the microscopic evaluation of all slides used for the study to maintain scientific rigor and quality. The results were not statistically different between the slides, repetitions, and scorers; therefore, results are presented as mean values for each donor.

\section{Quantification of apoptosis by caspase-3 activation}

Percent of late apoptotic cells in population was determined by caspase- 3 activation detection, which is the protease involved in the executive phase of this programmed cell death. Activation of caspase- 3 by lymphocytes was detected at 6 and $24 \mathrm{~h}$ after photon and proton irradiation with the use of caspase-3 DEVD-R110 Fluorometric HTS Assay Kit (Biotium, Hayward, CA, USA). This assay enables for a 
single-step high throughput screening (HTS) for caspase-3 enzyme activity (21).

Right after irradiation, lymphocytes in RPMI 1640 culture medium (PAA Laboratories GmbH, Pasching, Austria) were plated (black 96 well-plate, 50,000 cells suspended in $100 \mu \mathrm{L}$ of medium per well). Generally, 5 representative fields in well per dose, per time point were analysed. After 6 or $24 \mathrm{~h}$ after irradiation, $100 \mu \mathrm{L}$ of assay buffer [enzyme substrate (AcDEVD)2-R110 (2 mM) with cell lysis/assay buffer at a ratio of $50 \mu \mathrm{L}$ substrate per $1 \mathrm{~mL}$ buffer] was added directly to each well with cells and following plate was incubated at $37^{\circ} \mathrm{C}$. After $60 \mathrm{~min}$ of incubation, fluorescence was measured with $470 \mathrm{~nm}$ excitation and $520 \mathrm{~nm}$ emission (TECAN Group Ltd., Männedorf, Switzerland). Non-treated lymphocytes were subjected simultaneously to the caspase-3 DEVD-R110 Fluorometric HTS Assay as a biological control. As a positive control solution of R110 was used ( $80 \mu \mathrm{M}$ R110 was diluted in $20 \mu \mathrm{M}$ cell lysis buffer). Additionally, induced cells and caspase-3 inhibitor as an inhibitor control were used. The fluorescence intensity of the blank (negative control) was measured $(0 \mu \mathrm{M}$ R110) and it was subtracted from each fluorescence value to calculate relative fluorescence units (RFU).

\section{Statistical analysis}

Microsoft Office Excel 2013 was used to analyse all data. The OriginPro 2017 (OriginLab Co., Northampton, MA, USA) was used for the graphic representation of the results. For all experimental points mean values, standard deviation (SD) and variance (VAR) were calculated. To investigate if the results derived by repetitions, slides, and scorers were comparable, Pearson's correlation coefficient was used. For each data set Shapiro-Wilk normality test was performed, confirming normal distributions $(\mathrm{P}<0.05)$. Moreover, to test correlation between individual variability in apoptotic cells fraction (\%) obtained by Annexin V-FITC staining and age of each patient also Pearson's correlation coefficient was calculated. To evaluate the statistical significance of compared results three-sigma limit test was used $(\mathrm{P}<0.05)$.

\section{Results}

Quantitative determination of apoptosis in ex vivo HPBL model by Annexin V-FITC fluorescent staining after proton irradiation

The percentage of apoptotic cells was determined by the Annexin V-FITC fluorescent staining using Apoptotic,
Necrotic and Healthy Cells Quantification Kit (Biotium, Inc.) in HPBL obtained from 5 healthy donors after 1, 4, and $24 \mathrm{~h}$ of ex vivo irradiation with protons. Results for following doses of ionizing radiation: $0.3,0.5,0.75,1.0,1.5$, 2.0, 2.5, 3.0 and 4.0 Gy are presented separately for each donor in Figure 1. The results show an increase in apoptotic cells in Annexin V-FITC detection with dose, which peaked at 3.0 Gy after 1 and $4 \mathrm{~h}$ of irradiation. At $24 \mathrm{~h}$ postirradiation, percentage of apoptotic cells were similar in all subjects except in donor \#1 after 1.0 Gy. The percentage of Annexin V-FITC stained cells is significantly different $(\mathrm{P}<0.05)$ after 1 and $4 \mathrm{~h}$ vs. $24 \mathrm{~h}$ after irradiation.

Figure 1 shows percent apoptotic cells determined with Annexin V-FITC fluorescent staining in ex vivo HPBL model after $1 \mathrm{~h}$ (Figure 1A), $4 \mathrm{~h}$ (Figure 1B), and $24 \mathrm{~h}$ (Figure $1 C$ ) after proton irradiation for each donor at different radiation doses in the range 0-4.0 Gy. Average trend for all donors is shown with the grey line. The values; mean, SD, and VAR are provided in Table 1. For each donor, the number of cells was normalized to allow direct comparison. The mean apoptotic cells percentage increased significantly $(\mathrm{P}<0.05)$ with the dose up to 3.0 Gy. At 3.0 Gy dose, variability of apoptotic cells in HPBL population after proton irradiation peaked. Shapiro-Wilk normality test confirmed normal distributions $(\mathrm{P}<0.05)$ for each dataset. At $24 \mathrm{~h}$ post-irradiation, individual variability is more pronounced at $1.0 \mathrm{~Gy}$.

\section{Confirmation of apoptosis by caspase- 3 activation in HPBL after 6 and 24 hours of ex vivo proton irradiation}

In order to confirm apoptosis, we also measured caspase-3 activity, which is independent to necrotic mode of cellkilling, after 6 and 24 h of proton irradiation by using caspase-3 DEVD-R110 Fluorometric HTS Assay Kit (Biotium, Inc.). Active or cleaved caspase-3 is an indication of cells undergoing apoptosis as opposed to the Annexin $\mathrm{V}$ staining that can only reflect early apoptosis and cannot distinguish necroptotic cells. Results in the dose range 0.3 to 4.0 Gy as indicated by the fluorescent intensity representing caspase- 3 activity after 6 and $24 \mathrm{~h}$ of protons irradiation is presented separately for each donor in Figure 2 and Table 2. Mean fluorescence intensity after $6 \mathrm{~h}$ of proton irradiation is not statistically different between given doses in the studied dose-range (Figure 2, gray trend line). However, results suggest differences among individuals in caspase-3 activation (Figure 2A,B) with the highest variance value observed at the $0.5 \mathrm{~Gy}$. 

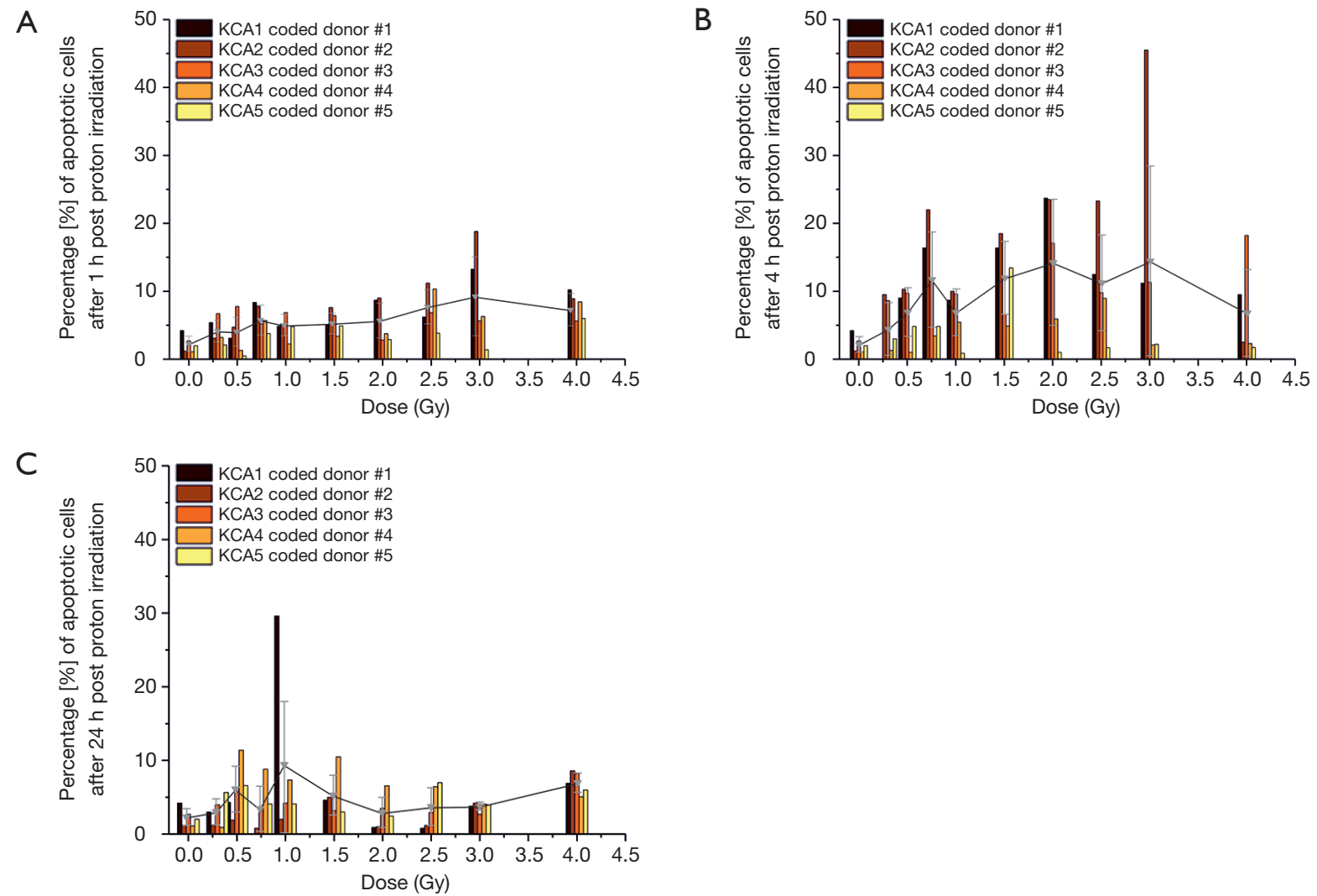

Figure 1 Apoptotic cells percent determined with Annexin V-FITC fluorescent staining in ex vivo HPBL model after $1 \mathrm{~h}$ (A), $4 \mathrm{~h}$ (B), and $24 \mathrm{~h}(\mathrm{C})$ after proton irradiation for each donor at different radiation doses in the range 0-4.0 Gy. Average trend for all donors is shown with the grey line. HPBL, human peripheral blood lymphocytes; FITC, fluorescein isothiocyanate.

Table 1 The values of mean, standard deviation (SD), and variance (VAR) of apoptotic cells in HPBL after ex vivo irradiation with protons in the dose range $0-4.0 \mathrm{~Gy}$

\begin{tabular}{|c|c|c|c|c|c|c|c|c|c|}
\hline Dose (Gy) & \multicolumn{3}{|c|}{$1 \mathrm{~h}$} & \multicolumn{3}{|c|}{$4 \mathrm{~h}$} & \multicolumn{3}{|c|}{$24 \mathrm{~h}$} \\
\hline 0 & 2.29 & 1.13 & 1.28 & 2.24 & 1.14 & 1.30 & 2.24 & 1.14 & 1.30 \\
\hline 0.3 & 4.18 & 1.60 & 2.56 & 4.48 & 3.86 & 14.91 & 2.95 & 1.78 & 3.15 \\
\hline 0.5 & 4.07 & 2.14 & 4.59 & 6.97 & 3.55 & 12.57 & 6.08 & 3.14 & 9.84 \\
\hline 1.0 & 5.07 & 1.58 & 2.50 & 6.92 & 3.42 & 11.72 & 9.45 & 7.22 & $104.43^{\#}$ \\
\hline 1.5 & 5.31 & 1.53 & 2.34 & 11.97 & 5.35 & $28.67^{\#}$ & 5.26 & 2.73 & 7.46 \\
\hline 2.0 & 5.74 & 2.59 & 6.73 & 14.25 & 9.25 & $85.57^{\#}$ & 2.87 & 2.07 & 4.30 \\
\hline 2.5 & 7.79 & 2.57 & 6.60 & 11.25 & 7.01 & $49.12^{\#}$ & 3.67 & 2.59 & 6.72 \\
\hline
\end{tabular}

", the highest dispersion within a group. VAR, variance calculated from all five healthy donors after 1,4 and $24 \mathrm{~h}$ post proton irradiation, respectively. HPBL, human peripheral blood lymphocytes. 

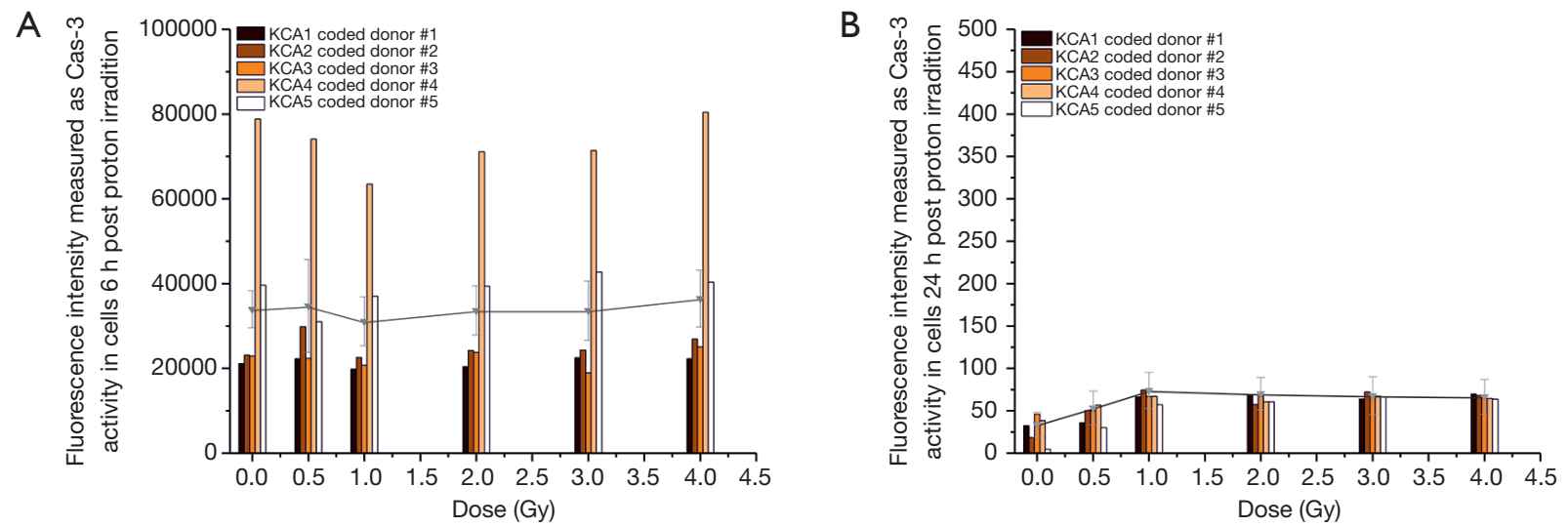

Figure 2 Differences in caspase- 3 activity among 5 donors at $6 \mathrm{~h}(\mathrm{~A})$ and $24 \mathrm{~h}(\mathrm{~B})$ after ex vivo irradiation of HPBL with protons in the dose range $0-4.0$ Gy. Gray trend line represents the average caspase-3 activity for the 5 donors. HPBL, human peripheral blood lymphocytes; FITC, fluorescein isothiocyanate.

Table 2 Caspase- 3 activity for all donors after 6 and $24 \mathrm{~h}$ after ex vivo proton irradiation of HPBL in the dose range 0-4.0 Gy

\begin{tabular}{|c|c|c|c|c|c|c|}
\hline Dose (Gy) & \multicolumn{3}{|c|}{$6 \mathrm{~h}$} & \multicolumn{3}{|c|}{$24 \mathrm{~h}$} \\
\hline 0 & $33,976.92$ & $4,383.20$ & $15,369,930.67$ & 33.52 & 14.64 & $1,071.07$ \\
\hline 1.0 & $31,115.04$ & $5,763.41$ & $26,573,552.21$ & 73.92 & 21.33 & $2,275.46$ \\
\hline 2.0 & $33,694.28$ & $5,815.69$ & $27,057,843.31$ & 69.99 & 19.13 & $1,829.54$ \\
\hline 4.0 & $36,504.60$ & $6,755.51$ & $36,509,516.18$ & 73.58 & 20.37 & $2,074.76$ \\
\hline
\end{tabular}

", the highest dispersion within a group. Mean fluorescence intensity, standard deviation (SD), variance (VAR) are calculated. HPBL, human peripheral blood lymphocytes.

Differences in caspase-3 activity among 5 donors at 6 and $24 \mathrm{~h}$ after $e x$ vivo irradiation of HPBL with protons in the dose range 0-4.0 Gy is shown in Figure 2. Gray trend line represents the average caspase- 3 activity for the 5 donors. Shapiro-Wilk normality test indicated a normal distribution within the donor groups $(\mathrm{P}<0.05, \mathrm{P}=0.37,0.91)$. Caspase- 3 activity is mostly observed at $6 \mathrm{~h}$ proton-irradiation, which decreased significantly after $24 \mathrm{~h}$ of irradiation $(\mathrm{P}<0.05)$. Moreover, the obtained results show statistical difference $(\mathrm{P}<0.05)$ for caspase- 3 activity between 6 and 24 h of irradiation. Results showed a difference in caspase- 3 activity among donors at $6 \mathrm{~h}$ post-irradiation, especially among donors \#4 and \#5, compared to donors \#1, \#2 and \#3 (Figure 2). Means, SD and VAR in fluorescence intensity representing caspase- 3 activity after 6 and 24 h following protons irradiation are presented in Table 2.
Inter-individual variability among donors in apoptosis frequency and caspase-3 activation in HPBL after proton irradiation

Individual variability in the frequency of apoptotic cells for each donor was determined by the Pearson correlation coefficient. The age of the donors was as follows: donor \#1, 40 years; donor \#2, 52 years; donor \#3, 56 years; donor \#4, 36 years; and donor \#5, 57 years. Despite the fact that determined correlation was not statistically strong $(\mathrm{P}<0.05$, $\mathrm{r}-\mathrm{PCC}=0.39$ ), Pearson correlation coefficient suggests that there might be a relationship in a larger group of donors.

\section{Discussion}

Use of proton therapy to improve local tumour control and minimize collateral normal tissue damage is rapidly 
emerging $(1-3,22)$. Principles of proton therapy related to energy deposition and advances in technology for precise delivery of dose that allows accurate tumour targeting has been previously reviewed (3). Similarly, current landscape of strategies to improve radiotherapy with an enhanced understanding of radiation-induced cellular damage have also been reviewed $(2,23,24)$. However, mechanistic studies designed to understand proton-irradiation induced cellkilling and their potential implications are relatively sparse. Therefore, here we aimed our studies on understanding the mechanisms of proton-irradiation induced apoptosis. The discussion below focuses on: (I) apoptosis in the ex vivo HPBL model as surrogate to study normal tissue injury; (II) role of caspase-3 activation in proton-irradiation induced apoptosis in HPBL; (III) inter-individual variation in a small cohort of healthy human donors using apoptosis cell frequency and caspase-3 activity as endpoints; (IV) HPBL irradiated ex vivo as model to estimate normal tissue relative biological effectiveness (RBE); (V) combining radiotherapy with cancer immunotherapy; (VI) and discuss potential implications of these studies in the context of precision radiation oncology.

Normal tissues are invariably included within the volume of treatment thus resulting in unavoidable acute, intermediate, and long-term adverse effects of radiation therapy (24). Circulating peripheral blood lymphocytes is a homogeneous cell population, in which cells are in the $G_{0}$ phase of the cell cycle. In this cell population, the process of cell-killing could be better studied compared to many other cell types in culture because confounding effects related to inhomogeneity of cell population, cell cycle phases, and any perturbations due to cell cycle arrest induced by irradiation do not interfere with measurements. Circulating lymphocytes represent normal tissue and traffic throughout the body including irradiated tumour volume. For example, radiation-induced micronucleus frequency in HPBL correlates with normal tissue damage in cervical cancer patients undergoing radiotherapy (25). Chromosomal aberrations in HPBL studied to determine radiation sensitivity among breast cancer patients indicate that there is a close relationship between radiation-induced cell-killing and chromosomal aberrations (26). Radiationinduced lymphocyte apoptosis in specific subpopulation of CD4 and CD8 T-lymphocytes can predict differences in late toxicity between individuals (27).

Radiation induces cell-killing via multiple mechanisms, but our studies here focus only on apoptosis. In early stages of apoptosis, changes occur at the cell membrane. One of these plasma membrane alterations is the translocation of PS from the inner side of membrane to outer layer, by which PS becomes exposed at the external surface of the cell (28), which serves to recognise dying cells for phagocytosis (29). PS in the outer cell membrane could be experimentally detected with the use of Annexin V (a human $36 \mathrm{kDa}$ singlechain calcium-binding protein), which has high affinity to PS and can be detected with fluorescently conjugated probes by flow cytometry (28). While the Annexin V-FITC assay, which is based on translocation of PS to the outer cell surface, has been well-described for detecting apoptosis $(13,30)$, translocation of PS to outer membrane can also occur during cell necrosis, especially during late necrosis (28). Further, a key difference between apoptosis and necrosis is that during the initial phase of apoptosis, cell membrane remains intact, but during necrosis there is loss of cell membrane integrity (28). Therefore, microscopic analysis is critical to distinguish early apoptosis from necroptosis. Hence, we used fluorescent microscopy by the Annexin V-FITC binding assay to quantify the frequency of apoptotic, necrotic and healthy cells; however, as it cannot distinguish from late necrosis from apoptosis, we also measured caspase- 3 activation in HPBL following ex vivo irradiation with protons to confirm apoptotic activity as extrinsic and intrinsic apoptotic cell death pathways intersect at caspase-3 (11).

It has been shown that radiation induces apoptosis in lymphocytes, which increases with time after exposure (13). The data presented in this paper demonstrate that apoptotic cells were still observed $24 \mathrm{~h}$ after proton irradiation (Tables 1,2). In our studies variation in spontaneous background apoptosis levels of individuals was observed, which ranged from $1.1 \%$ to $4.2 \%$ among individuals with an average $2.24 \% \pm 1.14 \%$ (Tables 1,2 ), which is in general agreement with literature (31).

Canonical apoptotic mechanism that results in cell-killing within a few hours of irradiation is mediated via induction of caspase activity (32). Caspases are crucial mediators of apoptosis, among them caspase- 3 is a frequently activated death protease, which catalyses the specific cleavage of many key cellular proteins and a key specific hallmark of apoptosis (33). Caspase-3 is a critical effector of caspase for apoptosis, which is responsible for cleaving proteins, including cytoskeleton and associated proteins, kinases and members of the Bcl-2 family of apoptosis related proteins (34). These studies have further shown that caspase- 3 gets activated as early as $2 \mathrm{~h}$ and its activity markedly increases after 4-6 h; for example, after X-ray irradiation in MOLT- 
4 leukemia cells (34). Based on these time-course kinetics, we chose two post-irradiation time points of 6 and $24 \mathrm{~h}$ after proton irradiation to study caspase- 3 activity. Our results confirm that proton irradiation results in apoptotic activity, which is mediated through caspase-3 (Figure $2 A, B$ ) at all doses confirming our microscopic observations. However, we do not rule out other modes of cell-killing, which includes necrosis especially with protons as we previously reported (9). Caspase-3 activation also shows differences among individuals in its activity at different radiation doses, similar to the observed quantitative differences with Annexin V-FITC binding (Figure 1A,B,C and Table 1). Prolonged incubation of HPBL ex vivo until $24 \mathrm{~h}$ after proton irradiation showed plateau in caspase-3 activity at doses above 1.0 Gy, but differences between individuals were still observed (Figure $2 A$ ).

Understanding the radiotherapy influence on activation cell death modalities such as apoptosis, may lead to the discovery of novel radiation-type specific biomarkers of injury, identification of novel cellular targets and allow development of protection, mitigation, and therapeutic approaches to address normal tissue injury. Stratification of patients according to the risk of toxicity could potentially guide modality selection or interventions to moderate this risk in high-risk individuals or allow intensification of therapy in low-risk individuals. The differences among individuals in their sensitivities to proton irradiation, which can be measured in the HPBL model with the Annexin V-FITC staining and/or caspase-3 activity, following $e x$ vivo irradiation. A significant difference from the mean for a given individual in apoptotic cell frequency or caspase-3 activity or outside the expected range, may reflect hypersensitivity to proton irradiation. This observation can be critical for those individuals whose lymphocytes are sensitive to apoptosis and can develop leucopenia adversely affecting the therapeutic benefit. This method could be also clinically useful to determine late occurring normal tissue injury. It has been previously reported that low apoptosis in HPBL is an excellent indicator of radiation sensitivity and a predictor of adverse late effects $(27,35)$. In a study that examined the correlation between the in vitro apoptotic response of lymphocytes to radiation and risk of developing late gastrointestinal and genitourinary toxicities from radiotherapy of prostate cancer indicated that patients with late toxicity showed a lower lymphocyte apoptotic response to radiation, compared to patients who did not develop late toxicities (35). Predictive tests are under development such as radiation-induced lymphocyte apoptosis assay for patients who are at risk of developing late complication. Such an identification of patients who do not have risk of radiation-induced late complications may benefit from dose escalation protocol (36). In consideration of the importance of caspase- 3 activity in radiation-induced apoptosis execution, caspase- 3 could be potentially used as a bioindicator of individual radiosensitivity, in lieu of radiation-induced lymphocyte apoptosis or chromosomal aberrations. Recently, a significant inter-individual variation in active caspase- 3 activity was reported in lymphocytes of healthy donors following ex vivo irradiation of peripheral blood samples with Co-60 gamma rays (37).

Our results indicate that proton irradiation-induced apoptosis may depend individual radiation sensitivity of lymphocytes (Tables 1 and 2, see variance values). The phenomenon of inter-individual differences in radiation sensitivity is already well-known. This variation in radiation response is mainly due to the individual's intrinsic cellular response to radiation. These individuals could have altered genetic factors regulating DNA repair processes and/or controlling apoptosis. From our studies it could be inferred that caspase- 3 activity may sufficiently reflect this difference. However, there could also be age-dependency for caspase activity as seen in a previous study, where peripheral blood samples from three donors of different ages were exposed to $300 \mathrm{kVp} \mathrm{X}$-rays or $138 \mathrm{MeV}$ protons $(0.2$ and $9.0 \mathrm{~Gy}$ dose), in which CD4 and CD8 T-lymphocytes showed an age-dependent sensitivity to radiation-induced apoptosis. Radiation-induced apoptosis was about 4 times higher in $\mathrm{CD}^{+}$lymphocytes from the youngest donor compared to the oldest donor and was about 2 times greater in $\mathrm{CD} 8^{+}$ T-lymphocytes, both after X-ray and proton exposures (38).

The concept of RBE is developed on basis of tumour cellkilling. Current RBE models assume a value 1.1 for protons and used in the clinic, which is likely an underestimate (22). One of the main limitation of using the RBE value 1.1 in the clinic is that even though they may provide a reasonable prediction of tumour control probability, they do not address normal tissue complication probability (39), nor there is any consensus on what endpoints are more relevant for determining normal tissue-specific RBE (40). In general, increase in linear energy transfer (LET) results in increase in apoptotic response (40-42), which is a concern for normal tissue injury during treatment. In addition, RBE values will also depend on factors, dose depth and LET distribution (41) and our understanding of LET effects on normal tissue injury is relatively sparse (40). Furthermore, treatment planning approaches do not consider differences in normal tissue 
sensitivity among individuals to protons (43). As circulating lymphocytes represent normal tissue and traffic in and out of tumour, this model may a good surrogate to directly measure normal tissue injury by measuring apoptosis or caspase- 3 activity and tailor proton therapy to individual patients.

Our studies may also be important in the context of developing therapeutic strategies based on modes of cell-killing and their possible relations to the immune consequences $(44,45)$. Role of lymphocytes in the cancer immunity cycle has been studied for many years (46). For combining radiotherapy with cancer immunotherapy, knowledge of the mechanisms of cellkilling by different radiation types is crucial for the success of a given therapeutic radiation type, which will help our understanding of contribution of a specific radiation-type in the development of systemic anti-tumour immunity (47). This will help selection of patient to a given type of radiation treatment and then individualize treatment and optimize the effectiveness of radiation therapy in combination with other modalities, i.e., in combination with chemotherapies, immune checkpoint-blocking agents, or immune-stimulating agents in a rational manner $(48,49)$. In this context, data from the present studies might be highly valuable in developing appropriate response models to proton therapy as HPBL model can serve as a surrogate of normal tissue response to treatment and may allow optimization of treatment planning at an individual patient level.

\section{Conclusions}

Proton therapy is likely to become the corner stone in the treatment of certain malignancies because of its perceived radiobiological superiority in dose delivery and distribution. However, studies on the biological mechanisms of cellkilling by proton irradiation are relatively few, scattered, often studied using cell cultures, or speculative, compared to studies with photons. Our results here show that the proton irradiation induces apoptosis in HPBL, which shows a dose-effect relationship after 1 and $4 \mathrm{~h}$ of irradiation. After $24 \mathrm{~h}$ post radiation exposure, the apoptotic fraction of cells represented a similar trend similar to the early time-points of 1 and $4 \mathrm{~h}$. Moreover, our results also show that caspase- 3 activation shows a peak value at $6 \mathrm{~h}$ post proton-irradiation among healthy donors. Further, our studies also point towards a difference in the response of peripheral blood lymphocytes, to protons, among different individuals. Here, we present studies that are aimed at providing a better understanding of the mechanisms of proton irradiationinduced apoptosis, which could be exploited to identify radiation hypersensitive individuals and exclude them from radiation treatment for better clinical outcome, while others could possibly benefit from a dose escalation. In addition, an understanding the effect of proton irradiation on the activation of apoptotic pathway in HPBL may lead to the discovery of novel biomarkers of radiation injury, allow stratification of patients based on a risk of toxicity, allow selection of a patient for a specific type of radiation treatment $v s$. other, and/or use of radioprotector or a mitigator to enhance therapeutic benefit.

\section{Acknowledgments}

The U.S. National Cancer Institute's (NCI) Radiation Research Program supported Dr. Prasanna's and Dr. Ahmed's assistance. The views expressed are those of the authors; no endorsement by NCI has been given or inferred. Authors gratefully acknowledge the donors for providing blood samples, technical staff for irradiation and dosimetry, "Diagnostics" company in Kraków, Poland for help in blood samples collection. Authors are also grateful for scientific support given by Professor Wojciech M. Kwiatek from Institute of Nuclear Physics Polish Academy of Sciences in Kraków, Poland.

Funding: Our research was partially supported by Grant DEC-2013/09/D/NZ7/00324 from the National Science Centre, and partially supported by Malopolska 1 Regional Operational Program Measure 5.1 Krakow Metropolitan Area as an important hub of the European Research Area for 2007-2013 — access to laboratory equipment.

\section{Footnote}

Conflicts of Interest: All authors have completed the ICMJE uniform disclosure form (available at http://dx.doi. org/10.21037/tcr.2018.06.14). MMA and PGSP serves as an unpaid editorial board member of Translational Cancer Research. The other authors have no conflicts of interest to declare.

Ethical Statement: The authors are accountable for all aspects of the work in ensuring that questions related to the accuracy or integrity of any part of the work are appropriately investigated and resolved. The study was conducted in accordance with the Declaration of Helsinki (as revised in 2013). Authors declare having approval from the 
human bioethical committee of the Regional Medical Board in Krakow approved the informed consent form used in this study (No. 124/KBL/OIL/2013). Prior to blood collection, informed consent was obtained from the donors.

Open Access Statement: This is an Open Access article distributed in accordance with the Creative Commons Attribution-NonCommercial-NoDerivs 4.0 International License (CC BY-NC-ND 4.0), which permits the noncommercial replication and distribution of the article with the strict proviso that no changes or edits are made and the original work is properly cited (including links to both the formal publication through the relevant DOI and the license). See: https://creativecommons.org/licenses/by-nc-nd/4.0/.

\section{References}

1. Le QT, Shirato H, Giaccia AJ, et al. Emerging Treatment Paradigms in Radiation Oncology. Clin Cancer Res 2015;21:3393-401.

2. Liauw SL, Connell PP, Weichselbaum RR. New paradigms and future challenges in radiation oncology: an update of biological targets and technology. Sci Transl Med 2013;5:173sr2.

3. Durante M, Orecchia R, Loeffler JS. Charged-particle therapy in cancer: clinical uses and future perspectives. Nat Rev Clin Oncol 2017;14:483-95.

4. Miszczyk J, Rawojc K, Panek A, et al. Response of human lymphocytes to proton radiation of $60 \mathrm{MeV}$ compared to $250 \mathrm{kV} \mathrm{X}$-rays by the cytokinesis-block micronucleus assay. Radiother Oncol 2015;115:128-34.

5. Schaue D, Micewicz ED, Ratikan JA, et al. Radiation and inflammation. Semin Radiat Oncol 2015;25:4-10.

6. Park B, Yee C, Lee KM. The effect of radiation on the immune response to cancers. Int J Mol Sci 2014;15:927-43.

7. Belloni P, Meschini R, Czene S, et al. Studies on radiationinduced apoptosis in G0 human lymphocytes. Int J Radiat Biol 2005;81:587-99.

8. Eriksson D, Stigbrand T. Radiation-induced cell death mechanisms. Tumour Biol 2010;31:363-72.

9. Miszczyk J, Rawojć K, Panek A, et al. Do protons and $\mathrm{X}$-rays induce cell-killing in human peripheral blood lymphocytes by different mechanisms? Clin Transl Radiat Oncol 2018;9:23-9.

10. Durante M, Brenner DJ, Formenti SC. Does Heavy Ion Therapy Work Through the Immune System? Int J Radiat Oncol Biol Phys 2016;96:934-6.

11. Taylor RC, Cullen SP, Martin SJ. Apoptosis: controlled demolition at the cellular level. Nat Rev Mol Cell Biol 2008;9:231-41.

12. Zhang G, Gurtu V, Kain SR, et al. Early detection of apoptosis using a fluorescent conjugate of annexin $\mathrm{V}$. Biotechniques 1997;23:525-31.

13. Wilkins RC, Kutzner BC, Truong M, et al. Analysis of radiation-induced apoptosis in human lymphocytes: flow cytometry using Annexin $\mathrm{V}$ and propidium iodide versus the neutral comet assay. Cytometry 2002;48:14-9.

14. Green D, Kroemer G. The central executioners of apoptosis: caspases or mitochondria? Trends Cell Biol 1998;8:267-71.

15. Cohen GM. Caspases: the executioners of apoptosis. Biochem J 1997;326:1-16.

16. Leist $M$, Jaattela $M$. Four deaths and a funeral: from caspases to alternative mechanisms. Nat Rev Mol Cell Biol 2001;2:589-98.

17. Broker LE, Kruyt FA, Giaccone G. Cell death independent of caspases: a review. Clin Cancer Res 2005;11:3155-62.

18. Dai C, Krantz SB. Interferon gamma induces upregulation and activation of caspases 1,3 , and 8 to produce apoptosis in human erythroid progenitor cells. Blood 1999;93:3309-16.

19. Slonina D, Biesaga B, Swakon J, et al. Relative biological effectiveness of the $60-\mathrm{MeV}$ therapeutic proton beam at the Institute of Nuclear Physics (IFJ PAN) in Krakow, Poland. Radiat Environ Biophys 2014;53:745-54.

20. Absorbed dose determination in external beam radiotherapy: An international code of practice for dosimetry based standards of absorbed dose to water. Tecnical Report Series, vol 398. IAEA, Vienna; 2000.

21. Yazihan N, Ataoglu H, Akcil E, et al. Midkine secretion protects Hep3B cells from cadmium induced cellular damage. World J Gastroenterol 2008;14:76-80.

22. Loeffler JS, Durante M. Charged particle therapy-optimization, challenges and future directions. Nat Rev Clin Oncol 2013;10:411-24.

23. Begg AC, Stewart FA, Vens C. Strategies to improve radiotherapy with targeted drugs. Nat Rev Cancer 2011;11:239-53.

24. Prasanna PG, Stone HB, Wong RS, et al. Normal tissue protection for improving radiotherapy: Where are the Gaps? Transl Cancer Res 2012;1:35-48.

25. Palyvoda O, Mukalov I, Polanska J, et al. Radiationinduced DNA damage and its repair in lymphocytes of patients with head and neck cancer and healthy donors. Anticancer Res 2002;22:1721-5.

26. Jones LA, Scott D, Cowan R, et al. Abnormal 
radiosensitivity of lymphocytes from breast cancer patients with excessive normal tissue damage after radiotherapy: chromosome aberrations after low dose-rate irradiation. Int J Radiat Biol 1995;67:519-28.

27. Ozsahin M, Crompton NE, Gourgou S, et al. CD4 and CD8 T-lymphocyte apoptosis can predict radiationinduced late toxicity: a prospective study in 399 patients. Clin Cancer Res 2005;11:7426-33.

28. Vermes I, Haanen C, Steffens-Nakken H, et al. A novel assay for apoptosis. Flow cytometric detection of phosphatidylserine expression on early apoptotic cells using fluorescein labelled Annexin V. J Immunol Methods 1995;184:39-51.

29. Fadok VA, Voelker DR, Campbell PA, et al. Exposure of phosphatidylserine on the surface of apoptotic lymphocytes triggers specific recognition and removal by macrophages. J Immunol 1992;148:2207-16.

30. Rieger AM, Nelson KL, Konowalchuk JD, et al. Modified annexin V/propidium iodide apoptosis assay for accurate assessment of cell death. J Vis Exp 2011. doi: $10.3791 / 2597$.

31. Schmitz A, Bayer J, Dechamps N, et al. Intrinsic susceptibility to radiation-induced apoptosis of human lymphocyte subpopulations. Int J Radiat Oncol Biol Phys 2003;57:769-78.

32. Lee KB, Kim KR, Huh TL, et al. Proton induces apoptosis of hypoxic tumor cells by the p53-dependent and p38/JNK MAPK signaling pathways. Int J Oncol 2008;33:1247-56.

33. Porter AG, Janicke RU. Emerging roles of caspase-3 in apoptosis. Cell Death Differ 1999;6:99-104.

34. Feng Y, Hu J, Xie D, et al. Subcellular localization of caspase-3 activation correlates with changes in apoptotic morphology in MOLT-4 leukemia cells exposed to X-ray irradiation. Int J Oncol 2005;27:699-704.

35. Schnarr K, Boreham D, Sathya J, et al. Radiation-induced lymphocyte apoptosis to predict radiation therapy late toxicity in prostate cancer patients. Int J Radiat Oncol Biol Phys 2009;74:1424-30.

36. Azria D, Betz M, Bourgier C, et al. Identifying patients at risk for late radiation-induced toxicity. Crit Rev Oncol

Cite this article as: Miszczyk J, Rawojć K, Borkowska AM, Panek A, Swakoń J, Gałaś A, Ahmed MM, Prasanna PG. Therapeutic proton irradiation results in apoptosis and caspase-3 activation in human peripheral blood lymphocytes. Transl Cancer Res 2018;7(4):879-889. doi: 10.21037/ tcr.2018.06.14
Hematol 2012;84 Suppl 1:e35-41.

37. Santos N, Silva RF, Pinto M, et al. Active caspase-3 expression levels as bioindicator of individual radiosensitivity. An Acad Bras Cienc 2017;89:649-59.

38. Radojcic M, Crompton NE. Age dependence of T-lymphocyte apoptosis induced by high-energy proton exposure. Radiat Environ Biophys 2001;40:131-5.

39. Tommasino F, Durante M. Proton radiobiology. Cancers (Basel) 2015;7:353-81.

40. Mohan R, Held KD, Story MD, et al. Proceedings of the National Cancer Institute Workshop on Charged Particle Radiobiology. Int J Radiat Oncol Biol Phys 2018;100:816-31.

41. Paganetti H. Relative biological effectiveness (RBE) values for proton beam therapy. Variations as a function of biological endpoint, dose, and linear energy transfer. Phys Med Biol 2014;59:R419-72.

42. Ristic-Fira AM, Todorovic DV, Koricanac LB, et al. Response of a human melanoma cell line to low and high ionizing radiation. Ann N Y Acad Sci 2007;1095:165-74.

43. Buchsbaum JC. Are Treatment Toxicity Issues in Particle Therapy a Clarion Call for Biologic Treatment Planning Overall? Int J Radiat Oncol Biol Phys 2017;97:1085-6.

44. Dewey WC, Ling CC, Meyn RE. Radiation-induced apoptosis: relevance to radiotherapy. Int J Radiat Oncol Biol Phys 1995;33:781-96.

45. Amaravadi RK, Thompson CB. The roles of therapyinduced autophagy and necrosis in cancer treatment. Clin Cancer Res 2007;13:7271-9.

46. Chen DS, Mellman I. Oncology meets immunology: the cancer-immunity cycle. Immunity 2013;39:1-10.

47. Formenti SC, Demaria S. Combining radiotherapy and cancer immunotherapy: a paradigm shift. J Natl Cancer Inst 2013;105:256-65.

48. Golden EB, Apetoh L. Radiotherapy and immunogenic cell death. Semin Radiat Oncol 2015;25:11-7.

49. Bellone M, Calcinotto A. Ways to enhance lymphocyte trafficking into tumors and fitness of tumor infiltrating lymphocytes. Front Oncol 2013;3:231. 